\title{
Contribuições do Apoio Matricial em Saúde Mental na Atenção Primária: Revisão Integrativa da Literatura
}

\author{
Aline Barros de Oliveira $^{I^{*}}$, Leonardo Silva da Costa ${ }^{2}$, Cláudia Daniele Barros Leite-Salgueiro ${ }^{3}$, \\ Valquíria Farias Bezerra Barbosa ${ }^{4}$, Robervam de Moura Pedroza ${ }^{5}$, Ana Carla Silva Alexandre ${ }^{6}$, \\ Luzineide Lobato ${ }^{7}$, João Bosco Caraciolo Batista Júnior ${ }^{8}$, Ellyda Layanny Aguiar da Silva ${ }^{9}$
}

\begin{abstract}
Resumo: Objetivo: Identificar na literatura as contribuições do apoio matricial em saúde mental na atenção primária à saúde. Metodologia: Trata-se de uma revisão integrativa da literatura, realizada no mês de abril de 2017 , na Biblioteca Virtual em Saúde através das bases de dados SCIELO, LILACS, MEDLINE e BDENF. Resultados: Foram selecionados 10 estudos para compor a amostra. Entre as principais contribuições do apoio matricial em saúde mental na atenção primária encontramos: o fortalecimento da dinâmica de trabalho multidisciplinar e multiprofissional; a melhora na organização do acesso do usuário ao serviço; a ampliação da tomada de decisão das equipes e corresponsabilização do cuidado e o fortalecimento do ideal de grupo terapêutico. Conclusão: Há necessidade de fortalecimento do apoio matricial para que haja uma maior adesão desta pratica por parte dos profissionais, uma vez que muitos consideram esta ferramenta como uma atribuição a mais e não, como um facilitador no processo de trabalho.
\end{abstract}

Descritores: Saúde Mental; Serviços de Saúde Mental; Atenção Primária à Saúde; Apoio Matricial.

\section{Contributions of Matricial Support in Mental Health in Primary Care: Integrating Review of Literature}

Abstract: Objective: To identify in the literature the contributions of matrix support in mental health in primary health care. Methodology: This is an integrative review of the literature, carried out in April 2017, in the Virtual Health Library through the databases SCIELO, LILACS, MEDLINE and BDENF. Results: We selected 10 studies to compose the sample. Among the main contributions of matrix support in mental health in primary care we find:

\footnotetext{
${ }^{1}$ Discente do curso de Bacharelado em Enfermagem do Instituto Federal de Educação, Ciência e Tecnologia (IFPE), Campus Pesqueira (PE), Brasil. E-mail: aline.olv@hotmail.com.

${ }^{2}$ Discente do curso de Bacharelado em Enfermagem do Instituto Federal de Educação, Ciência e Tecnologia (IFPE), Campus Pesqueira (PE), Brasil. E-mail: leonardosilva.12@hotmail.com.

${ }^{3}$ Psicóloga (UNICAP), Docente e Pesquisadora do Instituto Federal de Educação, Ciência e Tecnologia de Pernambuco (IFPE) - Campus Pesqueira - Departamento: Bacharelado em Enfermagem. Doutora em Psicologia Clínica pela Universidade Católica de Pernambuco (UNICAP), Mestra em Ciências da Saúde pela Universidade de Pernambuco (UPE), Pós graduada em Saúde Coletiva pela mesma Universidade. Recife (PE), Brasil. E-mail: claudia.leite@ pesqueira.ifpe.edu.br.

${ }^{4}$ Enfermeira (UFPE), Docente e Pesquisadora do Instituto Federal de Educação, Ciência e Tecnologia de Pernambuco (IFPE) - Campus Pesqueira - Curso: Bacharelado em Enfermagem. Doutora em Ciências Humanas pela Universidade Federal de Santa Catarina (UFSC), Mestre em Bioquímica e Fisiologia pela Universidade Federal de Pernambuco (UFPE), Pós graduada em Educação Profissional pela UFPE - Fiocruz. Caruaru (PE), Brasil. E-mail: valquiria@pesqueira.ifpe.edu.br.

${ }^{5}$ Enfermeiro (UEPB), Docente e Pesquisador do Instituto Federal de Educação, Ciência e Tecnologia de Pernambuco (IFPE) - Campus Pesqueira - Curso: Bacharelado em Enfermagem. Mestre em Saúde da Família pela Universidade Federal do Rio Grande do Norte (UFRN), Pós graduado em Gestão de Serviços e Sistemas de Saúde pela Fiocruz. Garanhuns (PE), Brasil. E-mail: robervam@pesqueira.ifpe.edu.br. ${ }^{6}$ Enfermeira (FENFA), Docente e Pesquisadora do Instituto Federal de Educação, Ciência e Tecnologia de Pernambuco (IFPE) - Campus Pesqueira - Curso: Bacharelado em Enfermagem. Mestre em Ciência da Saúde pelo Instituto Universitário Italiano de Rosário (IUNIR), Pós graduada em Unidade de Terapia Intensiva pelo Centro Universitário Internacional (UNINTER). Pesqueira (PE), Brasil. E-mail: ana.alexandre@pesqueira.ifpe.edu.br.

${ }^{7}$ Biológa (UFRN), Docente e Pesquisadora do Instituto Federal de Educação, Ciência e Tecnologia de Pernambuco (IFPE) - Campus Pesqueira. Mestra em Psicobiologia pela Universidade Federal do Rio Grande do Norte. Recife (PE), Brasil. E-mail: luzineidelobato@ gmail.com;

${ }^{8}$ Enfermeiro (AEB), Pós graduado em Unidade de Terapia Intensiva pelo Centro de Ensino, Pesquisa e Extensão (Cenpex). Sanharó (PE), Brasil. E-mail: jbcaraciolo@ hotmail.com;

${ }^{9}$ Discente do curso de Bacharelado em Enfermagem do Centro Universitário Mauricio de Nassau, Campus Graças, Recife (PE), Brasil. E-mail: ellyda.04@hotmail.com.

*Autora correspondente.
} 
the strengthening of multidisciplinary and multiprofessional work dynamics; the improvement in the organization of the user's access to the service; the expansion of team decision-making and joint responsibility for care and the strengthening of the ideal therapeutic group. Conclusion: There is a need to strengthen the matrix support so that there is a greater adherence of this practice on the part of professionals, since many consider this tool as an assignment to more and not as a facilitator in the work process.

Keywords: Mental Health; Mental Health Services; Primary Health Care; Matrix Support

\section{Introdução}

Em meados da década de 70, iniciaram no Brasil movimentos em prol da Reforma Psiquiátrica, que ganhou força a partir da $1^{\circ}$ Conferência Nacional em Saúde Mental (CNSM), ocorrida em 1987, na qual foram discutidos os modelos da atenção psiquiátrica brasileira, tendo como enfoque o modelo hospitalocêntrico ${ }^{1}$. Este modelo pode ser caracterizado pela lotação dos manicômios e o tratamento pautado nos maus tratos as pessoas com transtornos mentais ${ }^{2}$.

Na segunda CNSM, foi pensando um novo modelo de atenção as demandas em saúde mental, tendo como participantes desta conferência, profissionais, pacientes e seus familiares, montando assim um modelo de fortalecimento da tríade, pautada numa visão holística e humanizada. A realização das conferências favoreceram a construção da lei no 10.126, que dispõe sobre os direitos das pessoas com transtorno mental, e tem como objetivo principal a redução do número de indivíduos internados e do tempo de internação, como também propõe a participação das famílias e da comunidade na assistência em saúde mental ${ }^{(1-3)}$.

Com a mudança do modelo hospitalocêntrico para o modelo psicossocial, fez-se necessário também que os profissionais em saúde mental modificassem suas condutas em relação a acompanhamento dos usuários com transtorno mental, pois segundo Lavall ${ }^{4}$, a atenção psicossocial centra seu olhar no sujeito, observando as suas relações no cotidiano, para que dessa forma o profissional, auxilie no processo de reinserção do paciente com transtorno mental na sociedade, facilitando assim a construção de estratégias para a execução do acolhimento nos âmbitos da atenção à saúde mental ${ }^{4}$.

Tanto para a promoção da reinserção social como do cuidado em saúde mental, foi instituída a Rede de Atenção Psicossocial (RAPS) regulamentada pela Portaria No 3.088 de dezembro de 2011, para indivíduos com sofrimento ou transtorno mental e com necessidades 
resultantes do consumo de álcool, crack e outras drogas no âmbito do SUS, os objetivos gerais da RAPS constituem: expandir o acesso à atenção psicossocial de toda a população, promover laços dos indivíduos com transtornos mentais e com necessidades resultantes do consumo de álcool, crack e outras drogas e seus familiares aos pontos de atenção e garantir a articulação e integração entre os pontos de atenção territorial das redes de saúde, classificando o cuidado através do acolhimento, do acompanhamento contínuo e da atenção às urgências 5 .

De acordo com a Portaria no 3.088 , de 23 de dezembro de 2011 do Ministério da Saúde ${ }^{5}$, os seguintes serviços, são pontos de atenção da RAPS na atenção primária à saúde: Unidade Básica de Saúde (UBS) como porta de entrada da RAPS é responsável por desenvolver ações de promoção a saúde mental, prevenção e cuidado dos transtornos mentais, ações de redução de agravos e cuidado para indivíduos com necessidades resultantes do uso de álcool, crack e outras drogas, compartilhadas, sempre que necessário, com os outros pontos da rede. Equipes de Atenção Básica para populações em situações específicas que são as Equipes de Consultório de Rua e Equipe de apoio aos serviços do componente Atenção Residencial de Caráter Transitório, os Centros de Convivência e o Núcleo de Apoio à Saúde da Família (NASF).

No que tange à atenção secundária, têm-se os Centros de Atenção Psicossocial (CAPS), que atendem indivíduos com transtornos mentais graves e persistentes e com necessidades resultantes do uso de álcool, crack e outras drogas, que são organizados da seguinte forma CAPS I, CAPS II, CAPS III, CAPS AD e CAPS i (atende crianças e adolescentes), cada CAPS sendo de acordo com a quantidade de habitantes do município ou região. Na atenção de urgência e emergência: Serviço de Atendimento Móvel de Urgência (SAMU) e Unidade de Pronto Atendimento (UPA). A RAPS conta também com outros serviços como: serviços de atenção em regime residencial, dentre os quais comunidades terapêuticas, atenção hospitalar, estratégias de desinstitucionalização e reabilitação psicossocial ${ }^{5}$.

As ações de saúde mental na atenção primária à saúde devem seguir o modelo de redes de cuidado, com base territorial e atuação transversal com políticas específicas, buscando com isso o estabelecimento de vínculos e acolhimento. Essas ações devem ser fundamentadas tanto nos princípios do SUS como nos da Reforma Psiquiátrica ${ }^{6}$.

Desta forma, na atenção primária, a Estratégia Saúde da família (ESF) é considerada porta de entrada para o sistema, como também é referência à atenção em saúde mental. A ESF é um modelo de atenção que fortalece a ideia de saúde como um direito a cidadania, e deve 
proporcionar serviços mais resolutivos, integrais e humanizados, pautados na intersetorialidade e na participação da comunidade nas ações de saúde e no controle social ${ }^{6}$.

A atenção à saúde mental integra as responsabilidades da atenção primária, numa perspectiva de integralidade do cuidado, preservando-se os princípios e diretrizes da Política Nacional de Atenção Básica (PNAB) tais como: respeito aos direitos humanos, atenção humanizada, a lógica do território, a sistematização à saúde mental em rede, a intersetoralidade, a recuperação psicossocial, a multiprofissionalidade/interdisciplinar, a desistitucionalização, a prática da cidadania dos usuários e a liberdade entre os usuários e suas famílias ${ }^{7}$.

A ESF é uma importante aliada no cuidado aos indivíduos com transtornos mentais, pois grande parte dos usuários encaminhados aos serviços especializados não possuem uma demanda específica que confirme uma atenção especializada. Sendo assim, indivíduos com transtornos mentais leves podem ser cuidados e ter acompanhamento nas Unidades Básicas de Saúde (UBS). Neste sentido, há a necessidade de aproximar os serviços de saúde mental e da atenção primária, o apoio matricial ou matriciamento é uma das condutas adotada atualmente ${ }^{8}$.

$\mathrm{O}$ apoio matricial entre as equipes é definido como um método de trabalho que visa assegurar um cuidado especializado, tanto no nível assistencial como técnico-pedagógico. Sendo assim, pressupõe, um cuidado compartilhado entre a equipe de referência (profissionais da atenção primária à saúde) e as outras equipes de especialistas da RAPS com a missão de agregar conhecimentos à equipe de referência, contribuindo para que aumentem sua capacidade de resolver algumas problemáticas existentes. $\mathrm{O}$ matriciamento tem por objetivo dar suporte técnico às equipes, bem como instaurar a corresponsabilização 9 .

No processo de construção conjunta do projeto terapêutico entre duas equipes (a de referência e a de apoio matricial), profissionais de várias especialidades compartilham seu saber quando se deparam com a realidade exposta ${ }^{10}$.

Para a realização do apoio matricial são utilizados alguns instrumentos para auxiliarem no cuidado aos usuários, estes instrumentos são: o projeto terapêutico singular (PTS), a interconsulta, a consulta conjunta, a visita domiciliar conjunta, o contato a distância com o uso do telefone e outras tecnologias de comunicação, o genograma e o ecomapa ${ }^{10}$.

Com a utilização destes métodos pretende-se fornecer aos profissionais da atenção primária um maior conhecimento sobre a saúde mental, fazendo com que atuem como catalizadores do processo terapêutico, bem como o acesso aos serviços de saúde e a resolutividade dos casos atendidos ${ }^{10}$. 
O matriciamento é considerado uma estratégia relevante no que tange o cuidado integral aos pacientes com transtorno mental, deve ser considerado uma prática comum para os profissionais uma vez que os auxiliam e os ajudam na resolutividade dos casos, além de fortalecer o vínculo com profissionais de outros serviços. Diante do exposto justifica-se a relevância do estudo, em identificar na literatura, as contribuições do apoio matricial em saúde mental na atenção primária à saúde.

\section{Objetivo}

Identificar na literatura, as contribuições do apoio matricial em saúde mental na atenção primária à saúde.

\section{Métodos}

Com vistas atingir os objetivos propostos, foi selecionado o método de revisão integrativa da literatura. Este tipo de metodologia tem o intuito de reunir e sintetizar resultados de estudos sobre delimitado tema ou questão, de uma forma sistemática e ordenada, que contribui para aprofundar o conhecimento sobre a temática insvestigada ${ }^{11}$.

Para sua realização, foram seguidas as etapas que constituem uma revisão integrativa, representadas por: definição do problema (questão norteadora); estabelecimentos dos critérios de inclusão e exclusão; identificação dos estudos selecionados; análise dos estudos que constituem a amostra; apresentação da revisão/ síntese do conhecimento ${ }^{12}$.

A questão norteadora da pesquisa foi: Quais as contribuições do apoio matricial em saúde mental na atenção primária à saúde?

Para a construção do presente trabalho, foi feita a busca de artigos científicos publicados na BVS (Biblioteca Virtual em Saúde), através das bases de dados Scientific Electronic Library Online (SCIELO), Literatura Latino Americana e do Caribe em Ciências da Saúde (LILACS), Sistema Online de Busca e Análise de Literatura Médica (MEDLINE) e Base de Dados de Enfermagem (BDENF). Para a busca das publicações, foram utilizados os descritores indexados 
como Descritores em Ciências da Saúde (DeCS) Saúde Mental, Atenção Primária á Saúde e Apoio Matricial, cujo levantamento bibliográfico foi realizado durante o mês de abril de 2017.

Os critérios de inclusão estabelecidos para a amostra foram: publicações entre os anos de 2010 a 2017, no idioma português, texto completo disponível, publicados nas bases eletrônicas já mencionadas, que fizessem referência ao apoio matricial na atenção primária. Foram considerados como critérios de exclusão teses e dissertações, estudos não relacionados com a proposta do estudo e publicações repetidas em mais de uma base eletrônica.

A busca foi realizada a partir do cruzamento dos descritores com o uso do conector booleano "AND" e resultou em um total de 24 artigos, o detalhamento desta busca pode ser observado no fluxograma contido na figura 1.

Figura 1: Fluxograma das etapas da coleta dos artigos nas bases de dados.

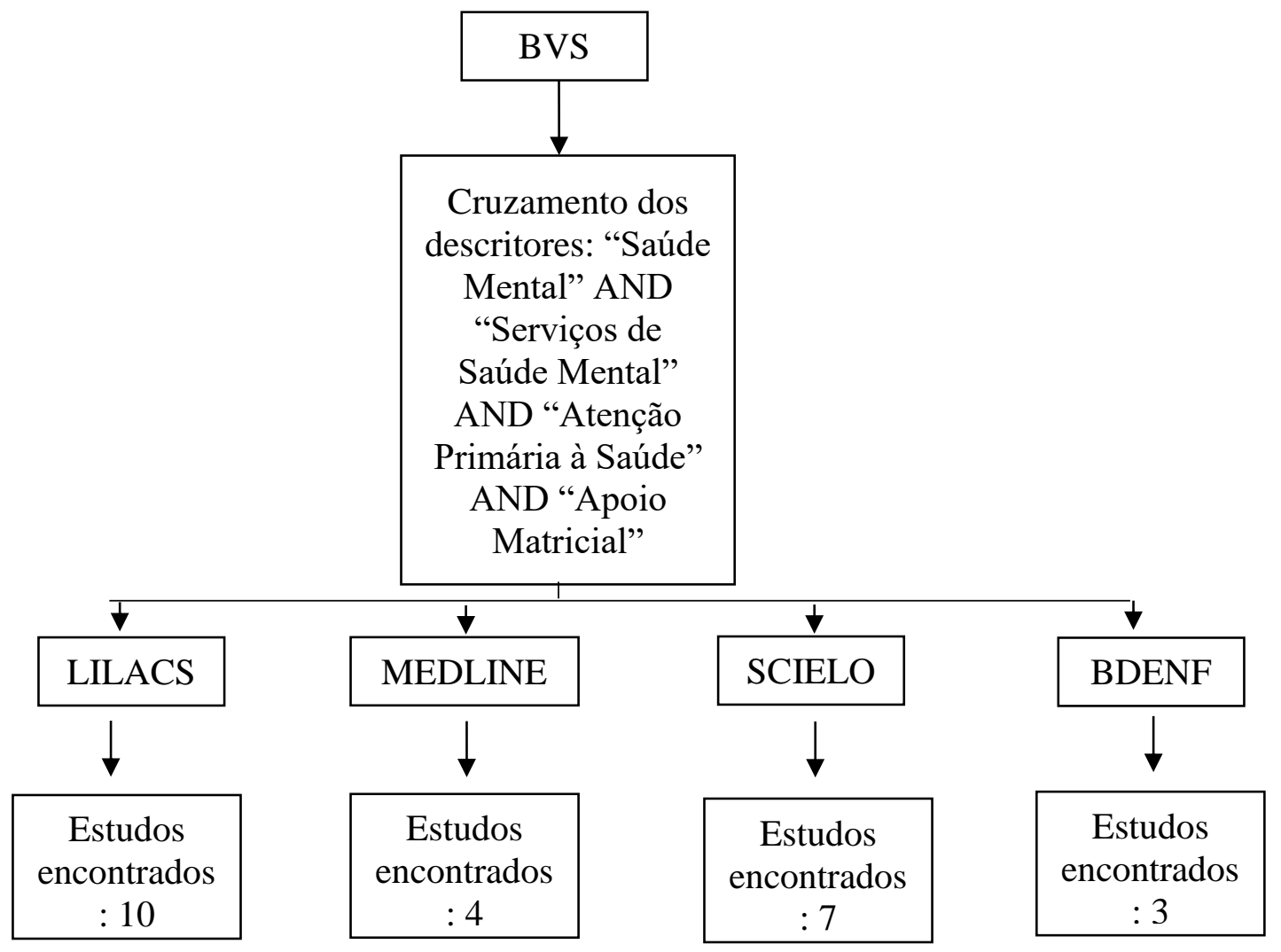

Fonte: Dados levantados pelos autores. 
Posteriormente, foram lidos os resumos e os que atenderam ao objetivo proposto e se encaixaram nos critérios de inclusão e exclusão foram lidos e relidos na íntegra, a partir disto, foram selecionados 10 estudos para subsidiar os resultados da pesquisa e possibilitar posterior catalogação em tabela. Os estudos foram selecionados através de um instrumento de coleta de dados adaptado de Ursi ${ }^{13}$ que continha: título, autores dos estudos, ano de publicação, tipo de estudo, base de dados, objetivo do estudo, local e região do Brasil em que o estudo foi publicado e principais contribuições do apoio matricial em saúde mental na atenção primária à saúde. De acordo com Ursi ${ }^{13}$ o objetivo deste instrumento é assegurar que os dados relevantes sejam extraídos em sua totalidade, minimizando o risco de erros na transcrição e garantindo a checagem das informações de forma precisa e segura. Os resultados obtidos da busca em cada base de dados e eliminados de acordo com os critérios de inclusão e exclusão estão presentes na tabela 1.

Tabela 1: Resultados obtidos nas bases de dados LILACS, Medline, Scielo e BDENF.

\begin{tabular}{|c|c|c|c|c|c|}
\hline Base de dados & $\begin{array}{c}\text { Resultados da } \\
\text { busca }\end{array}$ & $\begin{array}{c}\text { Estudos que não } \\
\text { atenderam o } \\
\text { objetivo proposto }\end{array}$ & $\begin{array}{c}\text { Estudos } \\
\text { indisponíveis }\end{array}$ & $\begin{array}{c}\text { Estudos } \\
\text { repetidos }\end{array}$ & $\begin{array}{c}\text { Estudos } \\
\text { selecionados }\end{array}$ \\
\hline LILACS & 10 & 2 & 1 & 2 & 5 \\
\hline MEDLINE & 4 & 1 & 1 & 1 & 1 \\
\hline SCIELO & 7 & 1 & 2 & 1 & 3 \\
\hline BDENF & 3 & 1 & 0 & 1 & 1 \\
\hline TOTAL & 24 & 4 & 5 & 3 & 10 \\
\hline
\end{tabular}

Fonte: Dados levantados pelos autores (2017).

\section{Resultados}

Foram selecionados a partir dos critérios estabelecidos, dez estudos que atenderam aos critérios de inclusão e aos objetivos propostos. A síntese do perfil dos estudos que integram a amostra, de acordo com código do artigo (estudo), título, autor, ano, abordagem metodológica e base de dados, está disposta no Quadro 1. 
Quadro 1. Quadro sinóptico dos estudos que compõem a amostra.

\begin{tabular}{|c|c|c|c|c|c|c|}
\hline Código & Autor (es) & & $\begin{array}{c}\text { Ano de } \\
\text { publica } \\
\text { ção }\end{array}$ & Título & $\begin{array}{l}\text { Abordagem } \\
\text { metodológica }\end{array}$ & $\begin{array}{c}\text { Base de } \\
\text { dados }\end{array}$ \\
\hline E1 & $\begin{array}{l}\text { QUINDERÉ, } \\
\text { JORGE, } \\
\text { NOGUEIRA, } \\
\text { COSTA, } \\
\text { VASCONCELOS, }\end{array}$ & $\begin{array}{l}\text { PHD; } \\
\text { MSB; } \\
\text { MSL; } \\
\text { LFA; } \\
\text { MGF }\end{array}$ & 2013 & $\begin{array}{l}\text { Acessibilidade e resolubilidade } \\
\text { da assistência em saúde } \\
\text { mental: a experiência do apoio } \\
\text { matricial }\end{array}$ & Qualitativo & LILACS \\
\hline
\end{tabular}

\begin{tabular}{|c|c|c|c|c|c|}
\hline E2 & $\begin{array}{l}\text { MORAIS, } \\
\text { TANAKA, OY }\end{array}$ & 2012 & $\begin{array}{l}\text { Apoio Matricial em Saúde } \\
\text { Mental: alcances e limites na } \\
\text { atenção básica }\end{array}$ & Qualitativo & LILACS \\
\hline E3 & $\begin{array}{l}\text { MINOZZO, F; COSTA, } \\
\text { II }\end{array}$ & 2013 & $\begin{array}{l}\text { Apoio matricial em saúde } \\
\text { mental entre CAPS e Saúde da } \\
\text { Família: trilhando caminhos } \\
\text { possíveis }\end{array}$ & Qualitativo & BDENF \\
\hline E4 & $\begin{array}{l}\text { PINTO, AGA; JORGE, } \\
\text { MSB; } \\
\text { VASCONCELOS, } \\
\text { MGF; SAMPAIO, JJC; } \\
\text { LIMA, GP; BASTOS, } \\
\text { VC; SAMPAIO, HAC }\end{array}$ & 2012 & $\begin{array}{l}\text { Apoio matricial como } \\
\text { dispositivo do cuidado em } \\
\text { saúde mental na atenção } \\
\text { primária: olhares múltiplos e } \\
\text { dispositivos } \\
\text { resolubilidade }\end{array}$ & Qualitativo & $\begin{array}{l}\text { MEDLI } \\
\mathrm{NE}\end{array}$ \\
\hline E5 & $\begin{array}{l}\text { ONOCKO-CAMPOS, } \\
\text { RT; CAMPOS, GWS; } \\
\text { FERRER, AL; } \\
\text { CORREA, CRS; } \\
\text { MADUREIRA, PR; } \\
\text { GAMA, CAP; } \\
\text { DANTAS, DV; } \\
\text { NASCIMENTO, R }\end{array}$ & 2012 & $\begin{array}{l}\text { Avaliação de estratégias } \\
\text { inovadoras na organização da } \\
\text { Atenção Primária à Saúde }\end{array}$ & Qualitativo & LILACS \\
\hline E6 & $\begin{array}{l}\text { RODRIGUES, ES; } \\
\text { MOREIRA, MIB }\end{array}$ & 2012 & $\begin{array}{l}\text { A Interlocução da Saúde } \\
\text { Mental com Atenção Básica } \\
\text { no Município de Vitoria/ES }\end{array}$ & Qualitativo & LILACS \\
\hline E7 & $\begin{array}{l}\text { VANNUCCHI, AMC; } \\
\text { JUNIOR, NC }\end{array}$ & 2012 & $\begin{array}{l}\text { Modelos tecnoassistenciais e } \\
\text { atuação do psiquiatra no } \\
\text { campo da atenção primária à } \\
\text { saúde no contexto atual do } \\
\text { sistema único de saúde, Brasil }\end{array}$ & Qualitativo & LILACS \\
\hline E8 & $\begin{array}{l}\text { NETO, MRG; } \\
\text { MEDINA, TSS; } \\
\text { HIRDES, A }\end{array}$ & 2014 & $\begin{array}{l}\text { Apoio matricial em saúde } \\
\text { mental na percepção dos } \\
\text { profissionais especialistas }\end{array}$ & Qualitativo & SCIELO \\
\hline E9 & $\begin{array}{l}\text { ONOCKO-CAMPOS, } \\
\text { R; GAMA, CA; } \\
\text { FERRER, AL, } \\
\text { SANTOS, DVD, } \\
\text { STEFANELLO,S; } \\
\text { TRAPÉ, TL; PORTO, K }\end{array}$ & 2011 & $\begin{array}{l}\text { Saúde mental na atenção } \\
\text { primária à saúde: estudo } \\
\text { avaliativo em uma grande } \\
\text { cidade brasileira }\end{array}$ & Qualitativo & SCIELO \\
\hline E10 & $\begin{array}{l}\text { ATHIÉ K, FORTES S, } \\
\text { DELGADO PGG }\end{array}$ & 2013 & $\begin{array}{l}\text { Matriciamento em saúde } \\
\text { mental na Atenção Primária: } \\
\text { uma revisão crítica (2000- } \\
\text { 2010) }\end{array}$ & Qualitativa & SCIELO \\
\hline
\end{tabular}

Fonte: Dados levantados pelos autores (2017). 
Dentre os estudos que compõem a amostra, 5 (50\%) correspondem ao ano de 2012, 3 (30\%) ao ano de 2013, já os anos de 2011 e 2014 apresentaram apenas 1 estudo (20\%), cada. No tocante a base de dados, 5 (50\%) foram encontrados na LILACS, 3 (30\%) na SCIELO, 1 (10\%) na BDENF, $1(10 \%)$ na MEDLINE.

No que se diz respeito a região geográfica de onde advém os estudos analisados, há considerável concentração de publicações nas regiões Sudeste e Sul, correspondendo a um percentual de $(60 \%)$ e (40\%) respectivamente, em contrapartida denota-se participação ainda discreta da região Nordeste.

Quanto à abordagem metodológica, observou-se totalidade de estudos de abordagem qualitativa, dado de extrema relevância.

No que concerne ao tipo de estudo, houve predomínio do estudo descritivo exploratório, o que converge para uma totalidade das publicações com nível de evidência IV.

As contribuições do apoio matricial para assistência em saúde mental por ser melhor visualizadas na Tabela 2.

Tabela 2. Contribuições do apoio matricial em saúde mental apontadas nos estudos que compõem a amostra.

\begin{tabular}{ccc}
\hline Contribuições & Frequência & Percentual Válido \\
\hline $\begin{array}{c}\text { Melhora na organização do } \\
\text { acesso do usuário ao serviço }\end{array}$ & 05 & $50 \%$ \\
\hline $\begin{array}{c}\text { Ampliação da tomada de } \\
\text { decisão das equipes }\end{array}$ & 04 & $40 \%$ \\
\hline $\begin{array}{c}\text { Fortalecimento do ideal de } \\
\text { grupo terapêutico para o } \\
\text { usuário }\end{array}$ & 02 & $20 \%$ \\
\hline $\begin{array}{c}\text { Uso de tecnologias leves como } \\
\text { alternativa de cuidado }\end{array}$ & 02 & $20 \%$ \\
\hline $\begin{array}{c}\text { Fortalecimento da dinâmica } \\
\text { de trabalho multidisciplinar } \\
\text { (Articulação entre dispositivos } \\
\text { da rede) }\end{array}$ & 07 & $70 \%$ \\
\hline $\begin{array}{c}\text { Corresponsabilização do } \\
\text { cuidado }\end{array}$ & 04 & $40 \%$ \\
\hline
\end{tabular}

Fonte: Elaborado pelos pesquisadores (2017).

Em meio os estudos que compõem a amostra, observa-se notável referência ao fortalecimento da dinâmica de trabalho multidisciplinar e multiprofissional (articulação entre 
dispositivos da rede), enquanto contribuição ofertada através da aplicação da lógica do apoio matricial, sendo este, referido em sete $(70 \%)$ dos artigos analisados.

Denota-se forte menção à melhora na organização do acesso do usuário ao serviço, tendo sido mencionada em cinco (50\%) dos estudos que compõem a amostra.

Houve referência à ampliação da tomada de decisão das equipes e à corresponsabilização do cuidado, ambas em quatro estudos (40\%), cada.

Outra contribuição do apoio matricial apontada nos estudos, foi o fortalecimento do ideal de grupo terapêutico para o usuário, apontado em dois (20\%) dos estudos.

O uso de tecnologias leves como alternativa de cuidado também foi apontado como contribuição do apoio matriciamento, mencionado em dois (20\%) dos estudos.

\section{Discussão}

A análise dos estudos que compõem a amostra, no que tange a observação da região geográfica de onde provém os mesmos, permitiu apontar uma apresentação que sugere um viés da iniquidade regional das publicações, bem como da oferta de serviços ${ }^{14}$.

O índice absoluto de estudos de caráter qualitativo na amostra em estudo é um ponto extremamente válido, tendo em vista seu alto potencial no que compete à compreensão dos fenômenos sociais e às nuances que permeiam o fazer em saúde ${ }^{15}$.

Neste estudo, quanto à força das evidências científicas dos estudos que compõem a amostra, ao considerar que as evidências científicas da Enfermagem possuem relevância, também, diante da sua potencialidade de respaldar o fazer e de associar as dimensões teóricas, práticas e científicas do cuidado, aponta-se a necessidade de desenvolverem-se novos estudos, com força de evidência maior nas produções de enfermagem, para assim, respaldar cientificamente as suas condutas, no tocante às contribuições do apoio matricial para o cuidado em saúde mental, na atenção primária à saúde ${ }^{16}$.

Em estudo, realizado por Machado e Camatta ${ }^{17}$, identificou-se por meio da análise da literatura nacional e internacional, contribuições e impasses, embora existam aspectos que comprometam a implantação do Apoio Matricial, sabe-se que, este, quando utilizado de maneira adequada, se apresenta como importante instrumento de interlocução entre os serviços que compõem a RAPS. 
A articulação do trabalho em equipe, que por sua vez é caracterizada como importante ferramenta no que se refere ao rompimento das práticas asilares, atuando assim como uma das formas de enfrentar a fragmentação do trabalho e do usuário, rumo à integralidade do cuidado, sendo este, fundamental no que tange a efetivação de uma lógica de atuação mais plural e integral por parte dos profissionais, bem como de uma multiplicidade de enfoques ${ }^{18}$.

Ainda no tocante a articulação em rede dos serviços de saúde, pode-se destacar a exímia contribuição advinda dos profissionais do CAPS no que se diz respeito a instrumentalização e o apoio às equipes da APS, no compete a atender as demandas emergidas pela população pela qual assumem responsabilidade, dessa forma o apoio que advém dos profissionais do CAPS, oportuniza a inserção do componente saúde mental atividades desenvolvidas no espaço comunitário, convergindo assim para um aumento da capacidade da equipe local em solucionar demandas em saúde mental ${ }^{19}$.

Entende-se que o apoio matricial em saúde mental na APS, pode vir a viabilizar a reorganização da rede de serviços, favorecer o acesso do usuário e ainda oportunizar o trânsito de trabalhadores do campo da saúde mental, anteriormente "confinados" aos CAPS, em detrimento dos mais variados níveis de complexidade do sistema de saúde ${ }^{8}$.

Dentro do presente contexto, é válido destacar que determinadas ações do apoio matricial poderão distinguir de maneira mais eficaz situações pontuais, individuais e sociais, que por ventura venham a justificar possíveis encaminhamentos para serviços especializados em saúde mental, além do mais, dada uma interação eficaz entre APS e o CAPS, as equipes de atenção primária se colocaram mais seguras no que compete a abordagem de casos de saúde mental, ampliando assim, a possiblidade de dar respostas às demandas dos usuários com transtornos mentais ${ }^{20}$.

No que tange ao fortalecimento dos mecanismos de tomada de decisão, à pluralidade de componentes na atuação profissional direcionada à assistência em saúde mental sob uma óptica matricial, garante maior apropriação na tomada de decisão destes profissionais, de forma que os mesmos não se limitam apenas à conduta clínica apontada por uma categoria profissional, mas sim, por todo um aparato multiprofissional e multidisciplinar ${ }^{21}$

No que se diz respeito a estratégia de corresponsabilização do cuidado, o uso este dispositivo, entre equipe e usuário incorpora na efetividade de sua dinâmica de trabalho, à família, à comunidade e às ações intersetoriais articuladas dentro de um contexto sócio comunitário enquanto ferramentas de apoio, desta forma, o diferencial do dispositivo 
terapêutico supracitado, se pauta no compartilhamento de problemas vividos e na pactuação integrada nas resoluções. Porém, o envolvimento compartilhado exige efetiva participação do usuário em seu processo de restabelecimento do equilíbrio ${ }^{22}$.

A análise da literatura permitiu identificar o matriciamento enquanto ferramenta que oportuniza o fortalecimento dos mecanismos de trabalho de grupos terapêuticos, acerca desse aspecto pode-se pontuar tais grupos, como percursores da descentralização em saúde mental, ao passo que tem como característica, a não obrigatoriedade da adscrição do usuário a determinada unidade básica de saúde, sendo tal preceito aderido, levando-se em consideração que alguns usuários não se sentem à vontade em grupos na sua comunidade de origem, por sentirem-se expostos, além do mais, deve-se considerar a oferta de múltiplas possibilidades de ampliar a rede social ${ }^{19}$.

Destaca-se ainda o uso de tecnologias leves como estratégia de cuidado fortalecida por meio do apoio matricial, entende-se que é possível desempenhar uma clínica solidificada em saberes e práticas inovadoras, a fim de abordar de modo complexo as demandas emanadas no usuário em relação a sua subjetividade, ferramentas de fácil execução, a exemplo da escuta qualificada, proporcionam o encontro entre a necessidade de se equilibrar psiquicamente e o interagir socialmente com maior grau de autonomia ${ }^{23}$.

\section{Conclusão}

Destaca-se que o matriciamento se apresenta enquanto ferramenta que oportuniza a reorganização de demandas em saúde mental na atenção primária a saúde, bem como sugere repensar o papel da reforma sanitária, sob a óptica da estratégia de saúde da família, bem como da reforma psiquiátrica, representada pelo CAPS.

A partir dos estudos analisados denotou-se concentração de publicações nas regiões Sul e Sudeste, e discreta participação das regiões Norte e Nordeste, a partir disto sugere-se a realização de novos estudos, a fim de disseminar maior número de informações acerca das contribuições do apoio matricial para o cuidado em saúde mental no âmbito da atenção primária a saúde. Além disso, obtivemos limitação também no que se refere a estudos quantitativos na temática abordada, tendo em vista o seu nível de evidência que é maior, outra lacuna encontrada foi na questão do quantitativo de material publicado no âmbito do apoio matricial, sendo assim 
recomenda-se a realização de novos estudos nessa temática e com níveis de evidência mais elevado, a fim de contribuir para a solidificação da enfermagem enquanto ciência como também para fortalecer a Prática Baseada em Evidências.

Conclui-se que há necessidade de fortalecimento do apoio matricial para que haja uma maior adesão desta pratica por parte dos profissionais, uma vez que muitos consideram esta ferramenta como uma atribuição a mais e não, como um facilitador no processo de trabalho.

\section{Referências}

1. Arantes EC, Stefanelli MC; Fakudo, IMK; Evolução histórica da enfermagem em saúde mental e psiquiátrica. In STEFANELLI, MC (org); Enfermagem Psiquiátrica e suas dimensões assistenciais. Monale. Brueri, São Paulo. 2008.

2. Borba LO, Guimarães NA, Mazza VA, Maftum MA.; Assistência em saúde mental sustentada no modelo psicossocial: narrativas de familiares e pessoas com transtorno mental RevEscEnferm USP, v. 48 n. 6, São Paulo. 2012.

3. Azevedo DM, Santos AT. Ações de Saúde Mental naAtenção Básica: Conhecimento de Enfermaeiros Sobre a Reforma Psiquiátrica. R. pesq.: cuid. Fundam. p. 3006-14, 2012.

4. Lavall E, Olschowsky A, Kantorski LP. Avaliação de família: rede de apoio social na atenção em saúde mental. Rev gaúcha enferm., v 30 n 2 pp. 198-205. Porto alegre, 2009.

5. Brasil. Ministério da Saúde. Portaria no 3.088, de 23 de dezembro de 2011. Brasília: Ministério da Saúde, 2011.

6. Junqueira MAB, Pillon SC. A assistência em saúde mental na Estratégia Saúde da Família: uma revisão de literatura. $R$. Enferm. Cent. O. Min. Minas Gerais, p. 260-267, 2011.

7. Brasil. Ministério da Saúde. Política Nacional de Atenção Básica. Brasília: Ministério da Saúde, 2011.

8. Quinderé PH, Jorge MSB, Nogueira MSL, Costa LFAC, Vasconcelos MGF. Acessibilidade e resolubilidade da assistência em saúde mental: a experiência do apoio matricial. Ciência \& Saúde Coletiva, 18(7):2157-2166, 2013.

9. Chiavagatti FG, Kantorski LP, Willrich JQ, Cortes JM, Jardim VMR, Rodrigues CGSS(2012). Articulação entre Centros de Atenção Psicossocial e Serviços de Atenção Básica de Saúde. Acta Paulista de Enfermagem, 25(1), 11-17. 
10. Brasil. Ministério da Saúde. Guia Prático de Matriciamento em Saúde Mental. Brasília: Ministério da saúde, 2011.

11. Mendes KDS, Silveira RCCP and Galvão, CM. Revisão integrativa: método de pesquisa para a incorporação de evidências na saúde e na enfermagem. Texto contexto - enferm. $2008 ; 17(4): 758-64$.

12. Botelho LLR, Cunha CCA, Macedo M. O método da revisão integrativa nos estudos organizacionais. Gestão e Sociedade. · Belo Horizonte, V. 5 • número 11. p. 121-136 • maio/agosto 2011.

13. Ursi ES. Prevenção de lesões de pele no perioperatório: revisão integrativa da literatura. [Internet]. Ribeirão Preto (SP): Universidade de São Paulo, Escola de Enfermagem de Ribeirão Preto; 2005.

14. Athié K, Fortes S, Delgado PGG. Matriciamento em saúde mental na Atenção Primária: uma revisão crítica (2000-2010). Rev Bras Med Fam Comunidade. Rio de Janeiro, 2013 Jan-Mar; 8(26):64-74.

15. Knauth DR, Leal AF. Expansion of the social sciences within public health: uses and abuses of qualitative research. Interface (Botucatu). 2014; 18(50):457-67.

16. Holanda ER; Lira MCC, Galvão MTG, Damasceno MMC, Araujo TL. Tendencies in the production of scientific knowledge in nursing regarding HIV/AIDS: a bibliometric study. 2013 Online braz j nurs [Internet].

17. Machado, D. K. S.; Camatta, M. W. Apoio Matricial como ferramenta de articulação entre a saúde mental e a atenção primária á saúde. Cad. Saúde Colet., Rio de Janeiro, v. 21, n. 2, p. 224-32, 2013.

18. Minozzo F, Costa II. Apoio matricial em saúde mental entre CAPS e Saúde da Família: trilhando caminhos possíveis. Psico-USF, Bragança Paulista, v. 18, n. 1, p. 151-160, jan./abril 2013.

19. Neto MRG, Medina STS, Hirdes MA. Apoio matricial em saúde mental na percepção dos profissionais especialistas. Aletheia 45, p.139-155, set./dez. 2014.

20. Travassos C, Martins M. Uma revisão sobre conceitos de acesso e utilização de serviços de saúde. Cad Saude Publica 2004; 20(Supl.2):190-198.

21. Morais APP, Tanaka OY. Apoio Matricial em Saúde Mental: alcances e limites na atenção básica. Saúde Soc. São Paulo, v.21, n.1, p.161-170, 2012.

22. Pinto AGA, Jorge MSB, Vasconcelos MG, Sampaio JJC, Lima GP, Bastos VC, Sampaio HAC et. al. Apoio matricial como dispositivo do cuidado em saúde mental na atenção primária: olhares múltiplos e dispositivos para resolubilidade. Ciência \& Saúde Coletiva, 17(3):653-660, 2012. 
23. Camuri D, Dimenstein M. Processos de trabalho em saúde: práticas de cuidado em saúde mental na Estratégia Saúde da Família. Saude Soc. 2010; 19(4): 803-81.

\section{Como citar este artigo (Formato ABNT):}

OLIVEIRA, Aline Barros de; COSTA, Leonardo Silva da; LEITE-SALGUEIRO, Cláudia Daniele Barros; BARBOSA, Valquíria Farias Bezerra; PEDROZA, Robervam de Moura; ALEXANDRE, Ana Carla Silva; LOBATO, Luzineide; BATISTA JÚNIOR, João Bosco Caraciolo; SILVA, Ellyda Layanny Aguiar da. Contribuições do Apoio Matricial em Saúde Mental na Atenção Primária: Revisão Integrativa da Literatura. Id on Line Rev.Mult. Psic., 2018, vol.12, n.41, p.1033-047. ISSN: 1981-1179.

Recebido: 11/07/2018.

Aceito: $26 / 07 / 2018$ 